\title{
NIVOLUMAB AS A WORSENING FATOR FOR PSORIATIC ARTHRITIS: A CASE REPORT
}

Viviane Ferreira de Vasconcelos ${ }^{1, \star}$, Laís Cristina Ferreira de Vasconcelos ${ }^{2}$, Karine Ferreira de Vasconcelos ${ }^{3}$, Luís Henrique Sarmento Tenório ${ }^{4}$, Daniela Aliny Tavares', Izabel Crystine Pereira Barbosa ${ }^{5}$, Guacyra Magalhães Pires Bezerra ${ }^{6}$

1.Universidade Federal de Pernambuco, Recife (PE), Brazil. 2.Universidade Católica de Pernambuco, Recife (PE), Brazil. 3.Hospital Militar de Área de Recife, Recife (PE), Brazil. 4.Hospital da Restauração, Recife (PE), Brazil. 5.Hospital Central da Aeronáutica, Recife (PE), Brazil. 6.Núcleo de Oncologia do Agreste, Caruaru (PE), Brazil.

*Corresponding author: vivi_ferreiravasc@hotmail.com

\section{BACKGROUND}

Psoriatic arthritis (PSA) is a systemic disease of an inflammatory nature, affecting joints and axial skeleton, entheses, skin and nails and is associated with a series of comorbidities and increased cardiovascular risk. The use of nivolumab may predispose to the onset or worsening of autoimmune diseases, including psoriatic arthritis, as a result of excessive activation of $\mathrm{T}$ cells and damage to normal tissues. A case of clinical worsening of the PsA is reported after the onset of nivolumab.

\section{CASE REPORT}

C.H.A., male, 73 years old, hypertense and ex-smoker. He reported being diagnosed with psoriasis in 2007 and shortly after the appearance of the cutaneous lesions, an inflammatory joint pain started - mainly in the knees, ankles, elbows and hands, with an increase in joint volume. He never was accompanied by a specialist and used only analgesic or anti-inflammatory when in crisis. Diagnosed with melanoma on the right foot in early 2020, he underwent surgery for tumor resection with subsequent onset of nivolumab for treatment. He reported worsening pain and joint swelling since starting this medication. On physical examination, he had arthritis in his wrists and first right metacarpophalangeal and telescope fingers. The case was discussed with the oncologist and was opted to start methotrexate and continue with nivolumab. The patient signed an informed consent form authorizing the case report.

\section{CONCLUSION}

Both rheumatologists and oncologists should be aware of the adverse musculoskeletal effects of nivolumab, as well as the chance of worsening existing rheumatological diseases. Immunotherapy is not contraindicated in patients with systemic autoimmune diseases; however, this patient will require greater care and follow-up, and sometimes therapy may have to be stopped in the face of serious adverse effects. 